\title{
ON SEMISIMPLE CLASSES OF ASSOCIATIVE AND ALTERNATIVE RINGS
}

\author{
by E. R. PUCZYŁOWSKI
}

(Received 3rd May, 1982)

In [6] Sands proved that the semisimple classes of associative rings are exactly the coinductive and closed under ideals and extensions classes. This characterization was transferred to the alternative case by Van Leeuwen, Roos and Wiegandt in [3]. Answering a question of [9], Sands [7] has recently proved that in the associative case the condition of being closed under ideals can be replaced by the regularity of the class. The same result for alternative rings has been proved by Anderson and Wiegandt in [2]. Thus the following result holds.

Theorem 1 ([2], [7]). A class $M$ of associative or alternative rings is semisimple if and only if $M$ is

(1) regular, that is, if $A \in M$ then every non-zero ideal of $A$ has a non-zero homomorphic image in $M$;

(2) coinductive, that is, whenever a ring $A$ contains a descending chain of ideals $B_{i}$ such that $\cap B_{i}=0$ and $A / B_{i} \in M$ for each $i$ then $A \in M$;

(3) closed under extensions, that is, $B \in M$ and $A / B \in M$ imply $A \in M$.

In the first part of this note we give a different proof of Theorem 1. Namely, we remark that this theorem is an almost immediate consequence of a technical result (probably a key-fact to all general theory of radicals of associative and alternative rings) proved by Terlikowska-Osłowska in [8].

Theorem 1 plays the decisive role in investigations of properties of semisimple classes. In Section 2 we present some results of this type.

In what follows we work in a universal class $W$ of alternative rings, that is, $W$ is a non-empty, hereditary to sub-rings and homomorphically closed class. All considered classes are supposed to be isomorphically closed and to contain the one-element ring $\{0\}$ subclasses of $W$.

For details of radical theory consult [10].

1.

To state the Terlikowska-Osłowska result required for the proof of Theorem 1 we need the following:

Definition (c.f. [8], Definition 2.1). An ideal $I$ of an alternative ring $A$ is said to be $q$-ideal if $A$ contains no ideals $L, K$ such that $L \subsetneq I \subsetneq K, K / I \approx I / L$ and $K^{2} \subseteq I$. 
Theorem 2. If $J$ is an ideal of an alternative ring $A$ and $I$ is a q-ideal of $J$ then $I$ is an ideal of $A$.

Proof. See Section 3 of [8].

Now we obtain Theorem 1 as a consequence of Theorem 2. Let $M$ be a subclass of $W$ satisfying the conditions (1)-(3) and let $U$ be the upper radical determined in $W$ by $M$. We will prove that $M$ is equal to the semisimple class of $U$. The condition (2) and Zorn's Lemma imply that any ring $A$ contains a minimal ideal $I$ such that $A / I \in M$. Let us denote such a minimal ideal $I$ by $A_{1}$ and let $A_{2}=\left(A_{1}\right)_{1}$. Minimality of $A_{1}$ and (3) imply that $A_{2}$ is an ideal of $A$ if and only if $A_{1}=A_{2}$. But if $U(A)=0$ and $A_{1} \neq 0$ then $A_{1}$ can be homomorphically mapped onto a non-zero ring in $M$, so $A_{2} \neq A_{1}$. Hence to show that $U(A)=0$ implies $A \in M$ or, equivalently, $A_{1}=0$ it is enough to prove that $A_{2}$ is an ideal of $A$. This condition is obviously satisfied for zero-rings, so zero-rings are $U$-semisimple if and only if they belong to $M$. Generally, in view of Theorem 2 , it suffices to prove that $A_{2}$ is a $q$-ideal of $A_{1}$. Let $K, L$ be ideals of $A_{1}$ such that $L \subsetneq A_{2} \subsetneq K$, $A_{2} / L \approx K / A_{2}$ and $K^{2} \subseteq A_{2}$. Now $U\left(K / A_{2}\right)=0$ as $K / A_{2}$ is an ideal of $A_{1} / A_{2} \in M$ and all rings of $M$ are $U$-semisimple. But $\left(K / A_{2}\right)^{2}=0$ so $K / A_{2} \in M$. Thus $A_{2} / L \in M$ too and by (3) $A_{1} / L \in M$. This contradicts minimality of $A_{2}$.

Remark. In our proof, besides Theorem 1 we used the Anderson-Divinsky-Suliński [1] theorem asserting that the semisimple classes are closed under ideals. Let us observe that to prove Theorem 2 in the associative case it is also enough to use the trick of their proof. Namely, if $I$ is not an ideal of $A$ then for some $a \in A, a I \not \subset I$ or $I a \not \subset I$. Let us assume that $a I \not \subset I$. If the ring $A$ is associative then $K=a I+I$ and $L=\{x \in I \mid a x \in I\}$ are ideals of $J$ and $(a I+I)^{2} \subseteq I$. Also $I / L \approx K / I$ by the mapping $f(i+L)=a i+I$. Hence $I$ cannot be a $q$-ideal of $J$.

2.

For any class $M \subseteq W$, let $\bar{M}$ denote the semisimple closure of $M$, i.e. the smallest semisimple class of $W$ containing $M$. In [6], applying his characterization of semisimple classes, Sands proved that the semisimple closure of a left strong class of associative rings is left strong too. Using Theorem 1 we can show a wider class of properties of rings inherited by the semisimple closure. In particular we obtain results like the ones Rossa and Tangeman did in [5] concerning lower radicals.

A relation $\sigma$ on $W$ will be called an $H$-relation (cf. [5]) if $\sigma$ satisfies the following conditions:

(a) $I \sigma R$ imples $I$ is a subring of $R$;

(b) $I \sigma R$ whenever $I$ is an ideal of $R$;

(c) if $I \sigma R$ and $f$ is a homomorphism on $R$ then $f(I) \sigma f(R)$;

(d) if $I \sigma R$ and $J$ is an ideal of $R$ then $I \cap J \sigma J$.

Examples of $H$-relations are "subring of", "left ideal of" and "ideal of". Other examples of $\boldsymbol{H}$-relations are discussed in [5]. 
If $I \sigma R$ then we say that $I$ is a $\sigma$-subring of $R$. A class $M \subseteq W$ is said to be $\sigma$-hereditary ( $\sigma$-regular) if any $\sigma$-subring of a ring from $M$ is in $M$ (any non-zero $\sigma$-subring of a ring from $M$ can be homomorphically mapped onto a non-zero ring in $M$ ).

The condition (b) guarantees that any $\sigma$-regular class is regular.

It can be easily checked that if $\sigma$ is an $H$-relation then so is $\bar{\sigma}=\{(I, A) \mid$ there exist subrings $I=I_{0} \subseteq \cdots \subseteq I_{n}=A$ such that $I_{i-1} \sigma I_{i}$ for $\left.i=1, \ldots, n\right\}$.

Clearly $M$ is $\sigma$-hereditary if and only if $M$ is $\bar{\sigma}$-hereditary.

Proposition 1. Any semisimple $\bar{\sigma}$-regular class $M \subseteq W$ is $\sigma$-hereditary.

Proof. Let $U \subseteq W$ be the upper radical determined by $M, A \in M$ and $I \sigma A$. Since $U(I)$ is an ideal of $I$ then $U(I) \bar{\sigma} A$. But $M$ is $\bar{\sigma}$-regular so if $U(I) \neq 0$ then some non-zero homomorphic image $J$ of $U(I)$ is in $M$. This is impossible as $J \in U$ and all rings of $M$ are $U$-semisimple. Thus $U(I)=0$ and, since $M$ is the semisimple class of $U, I \in M$.

For a class $M \subseteq W$, let $E M=\{A \mid A$ contains an ideal $I$ such that $I \in M$ and $A / I \in M\}$ and $P M=\{A \mid A$ is a subdirect sum of rings of $M\}$.

The following proposition is straightforward.

Proposition 2. (a) The union of a collection of $\sigma$-regular classes is a $\sigma$-regular class. In particular any class $M \subseteq W$ contains the largest $\sigma$-regular class $\sigma(M)$;

(b) If $M$ is a $\sigma$-regular class then so are EM and PM.

Proposition 2 and Theorem 1 imply immediately

Corollary 1. If $M \subseteq W$ is a semisimple class then so is $\sigma(M)$.

Corollary 2. If a class $M \subseteq W$ is $\sigma$-regular ( $\sigma$-hereditary) then so is $\bar{M}$.

Proof. If the class $M$ is $\sigma$-regular then $M \subseteq \sigma(\bar{M}) \subseteq \bar{M}$. By Corollary $1 \sigma(\bar{M})$ is a semisimple class. Since $\bar{M}$ is the smallest semisimple class containing $M$ then $\sigma(\bar{M})=\bar{M}$. Thus $\bar{M}$ is $\sigma$-regular.

Now let $M$ be $\sigma$-hereditary. Obviously $M$ is $\bar{\sigma}$-regular. As in the preceding paragraph $M$ is $\bar{\sigma}$-regular. But then Proposition 1 implies that $M$ is $\sigma$-hereditary. The result follows.

We say that a radical $S \subseteq W$ is a $\sigma$-radical if any $S$-radical $\sigma$-subring of a ring $A \in W$ is contained in $S(A)$. $\sigma$-radicals are related to $D$-radicals defined in [4]. Namely, if $I \sigma A$ and $I^{*}$ is the ideal of $A$ generated by $I$ then the condition (c) implies $I \sigma I^{*}$. Thus any $\sigma$ radical is a $D$-radical.

From Corollary 2 we obtain

Corollary 3. If $M \subseteq W$ is a regular class then the upper radical $U_{M} \subseteq W$ determined by $M$ is a $\sigma$-radical if and only if $M$ is $\sigma$-regular.

Using Theorem 1 we can prove that some natural constructions of classes of rings inherit semisimplicity. We present now some examples of such constructions.

For a ring $A$, let $A^{0}$ denote the zero ring on the additive group of $A$. For any class $M \subseteq W$ define $M^{0}=\left\{A \in W \mid A^{0} \in M\right\}$. Theorem 1, or the characterization of the semisimple classes given in [3] yields immediately 
Proposition 3. If $M \subseteq W$ is a semisimple class then so is $M^{0}$.

By comparison with Corollary 2, we obtain

Corollary 4. If $M \subseteq W$ is a class such that $M \subseteq M^{0}$ then $\bar{M} \subseteq \bar{M}^{0}$.

In what follows $W$ will be the class of all associative rings.

Let $F$ be a ring free as $\mathbb{Z}$-module, where $\mathbb{Z}$ is the ring of integers. As in Proposition 3 and Corollary 4 , we obtain similarly

Proposition 4. (a) if $M$ is a semisimple class then so is $F M=\left\{A \mid A \bigotimes_{\mathrm{Z}} F \in M\right\}$.

(b) if $M \subseteq F M$ then $\bar{M} \subseteq F \bar{M}$.

Proposition 5. For any semisimple class $M$ of rings the class $M_{1}=\{R \mid$ any non-zero left ideal of $R$ can be homomorphically mapped onto a non-zero ring in $M\}$ is semisimple.

Proof. It is easy to check that $M_{1}$ is subdirectly closed and closed under extensions. To prove that the class $M_{1}$ is semisimple it is enough to show that $M_{1}$ is closed under ideals. Let $I$ be an ideal of $A \in M_{1}$ and $L$ a non-zero left ideal of $I$. Then $L+A L$ is a non-zero left ideal of $A$ and $L$ is an ideal of $L+A L$. Also $(L+A L)^{2} \subseteq L$. Since $A \in M_{1}$ then $L+A L$ can be homomorphically mapped onto a non-zero ring $B \in M$. Let $f: L+A L \rightarrow B$ be such a homomorphism. If $L \nsubseteq \operatorname{Ker} f$ then $f(L)$ is a non-zero ideal of $B$. But the class $M$ is regular so $f(L)$, and hence also $L$, can be homomorphically mapped onto a non-zero ring in $M$. Thus let $L \subseteq \operatorname{Ker} f$. Then $B^{2}=0$ and $B=\sum_{a \in A} f(a L)$. Now it is easy to see that for any $a \in A$ the mapping $g: L \rightarrow f(a L)$ given by $g(l)=f(a l)$ is a ring homomorphism. For some $a \in A, f(a L) \neq 0$. Certainly $f(a L)$ is an ideal of $B$ as $B^{2}=0$. Thus $f(a L)$, and hence also $L$, can be homomorphically mapped onto a non-zero ring in $M$. This ends the proof.

Remark. For any $\boldsymbol{H}$-relation $\sigma$ and any class $\boldsymbol{M}$ of alternative rings we can define $M_{1}=\{A \in M \mid$ any non-zero $\sigma$-subring of $A$ has a non-zero homomorphic image in $M\}$ and for $n \geqq 2, M_{n}=\left(M_{n-1}\right)_{1}$. It can be easily checked that if the class $M$ is semisimple then $\sigma(M)=\bigcap_{n=1}^{\infty} M_{n}$. This fact can be used to obtain Corollary 2, though it is still unclear how many steps are necessary. It seems to be possible that in the case when $\sigma=$ "left ideal of" the construction stops after a finite number of steps.

\section{REFERENCES}

1. T. Anderson, N. Divinsky and A. Sulinsski, Hereditary radicals in associative and alternative rings, Canad. J. Math. 17 (1965), 594-603.

2. T. Anderson and R. Wiegandt, Semisimple classes of alternative rings, Proc. Edinburgh Math. Soc. 25 (1982), 21-26.

3. L. C. A. Van Leeuwen, C. Roos and R. Wiegand, Characterizations of semisimple classes, $J$. Austral. Math. Soc. (Series A) 23 (1977), 172-182.

4. E. R. PuczyŁowski, Radicals of rings and their subrings, Proc. Edinburgh Math. Soc. 24 (1981), 209-215.

5. R. F. Rossa and R. L. Tangeman, General heredity for radical theory, Proc. Edinburgh Math. Soc. 20 (1976/77), 333-337. 
6. A. D. SANDS, Strong upper radicals, Quart. J. Math. (Oxford) 27 (1976), 21-24.

7. A. D. SANDs, A characterization of semisimple classes, Proc. Edinburgh Math. Soc. 24 (1981), 5-7.

8. B. Terlikowska-Ostowsra, Category with a self-dual set of axioms, Bull. Acad. Polon. Sci., Ser. Sci. Math. Astronom. Phys. 25 (1977), 1207-1214.

9. R. WiEgandt, List of Problems, Kolloquium uber Algebra (Vienna, 1978), 6.

10. R. WIEgandt, Radical and semisimple classes of rings, (Queen's Papers in Pure and Applied Mathematics, No. 37, Kingston, Ontario, 1974).

Institute of Mathematics

UNIVERSTTY

PKiN, 00-901 WARSAW 